\title{
Hemodynamic Effects of Local Anesthesia and its Possible Correlation with Chromogranin A
}

\author{
Maha T. Al-Saffar ${ }^{1}$, Tahani A. AL-Sandook ${ }^{2}$, Mahmoud Y. Taha ${ }^{1, *}$ \\ ${ }^{1}$ Department of Dental Basic Science, College of Dentistry, Mosul University, Mosul, Iraq \\ ${ }^{2}$ Iraq Cultural Attachi, Washington, USA \\ *Corresponding author: tahadent04@yahoo.com
}

Received December 30, 2013; Revised January 20, 2014; Accepted January 22, 2014

\begin{abstract}
Aim: To study the effects of local anesthesia on blood pressure in patients undergo extraction of maxillary teeth under infiltration local anesthesia and to compare the level of salivary chromogranin A before and after administration of local anesthesia and its possible correlation with hemodynamic effects of local anesthesia. Materials and Methods: A total of 26 patients (18 female, 8 male) who need tooth extraction under infiltration local anesthesia were examined in two situations (pre and post local anesthetic administration). For each patient salivary sample was collected by salivette to estimate the level of chromogranin A using ELISA Kits. For all patients, the blood pressure and pulse rate were measured before and after administration of local anesthesia using automatic blood pressure recorder. Results: the results showed significant differences between salivary chromogranin A for patients before the administration of local anesthesia $(580.33 \pm 130.42) \mathrm{ng} / \mathrm{ml}$ and after the administration of local anesthesia $(674.51+130.93) \mathrm{ng} / \mathrm{ml}$ with $\mathrm{p}<0.05$. The results also showed that the mean value of blood pressure (systolic and diastolic blood pressure) lowered after administration of local anesthesia but with no significant differences $(p>0.05)$ with pre -local anesthetic value. The pulse rate was increase in it is value after local anesthetic administration with a significant differences $(\mathrm{p}<0.05)$ than pre - anesthetic value. Conclusions: tooth extraction and administration of infiltration local anesthesia caused hypotension with no significant effect $(p>0.05)$ while it caused increase in pulse rate with a significant effect $(\mathrm{p}<0.05)$. Administration of infiltration local anesthesia caused significant effects on salivary chromogranin A levels, also this study showed that there was significant inverse correlation between systolic blood pressure and chromogranin A levels $(\mathrm{p}<0.05)$ after administration of infiltrations local anesthesia during dental treatments (tooth extraction).
\end{abstract}

Keywords: salivary chromogranin A, local anesthesia, blood pressure

Cite This Article: Maha T. Al-Saffar, Tahani A. AL-Sandook, and Mahmoud Y. Taha, "Hemodynamic Effects of Local Anesthesia and its Possible Correlation with Chromogranin A." American Journal of Pharmacological Sciences, vol. 2, no. 1 (2014): 12-17. doi: 10.12691/ajps-2-1-3.

\section{Introduction}

LA is a temporary loss of sensation including pain in one part of the body produced by a topically applied or injected agent without depressing the level of consciousness. [1] Dental anxiety and fear of pain associated with dentistry are relatively stable over time. Despite advances in dental equipment, procedure and preventive measures, dental anxiety is a confounding problem with which dentist has to cope [2].

Salivary chromogranin A was shown to be biomarker of acute stress. It was the first granin to be isolated and characterized as uniquely acidic protein co stored and co released with the catecholamine hormones from the bovine adrenal medulla. [3] It was recently demonstrated that chromogranin $\mathrm{A}$ and $\mathrm{B}$ each regulates the concentration of adrenalin in chromaffin granules and its exocytosis. [4] In contrast to catecholamines which are removed relatively quickly from the circulation by cellular uptake or catabolism. Circulating CgA is relatively stable, so it has therefore been proposed as a useful marker of sympathetic nervous system activity. [5,6] CgA is expressed and released from serous acinar and ductal cells of the human submandibular salivary gland [7].

The relationship between mean arterial blood pressure (MAP), Cardiac output (CO) and total peripheral resistance (TPR) can be described in Figure 1 and equation.

$$
\mathrm{MAP}=\mathrm{CO} \text { TRP }
$$

Aim: to study the effects of local anesthesia on blood pressure in patients undergo extraction of maxillary teeth under infiltration local anesthesia and to compare the level of salivary chromogranin A before and after administration of local anesthesia and a possible correlation with hemodynamic effects of local anesthesia.

\section{Patients, Materials and Methods}

This study was carried out at the specialized center for dentistry in Mosul city / Iraq, from February 2013 to July 


\section{Drivers of Arterial Blood Pressure}

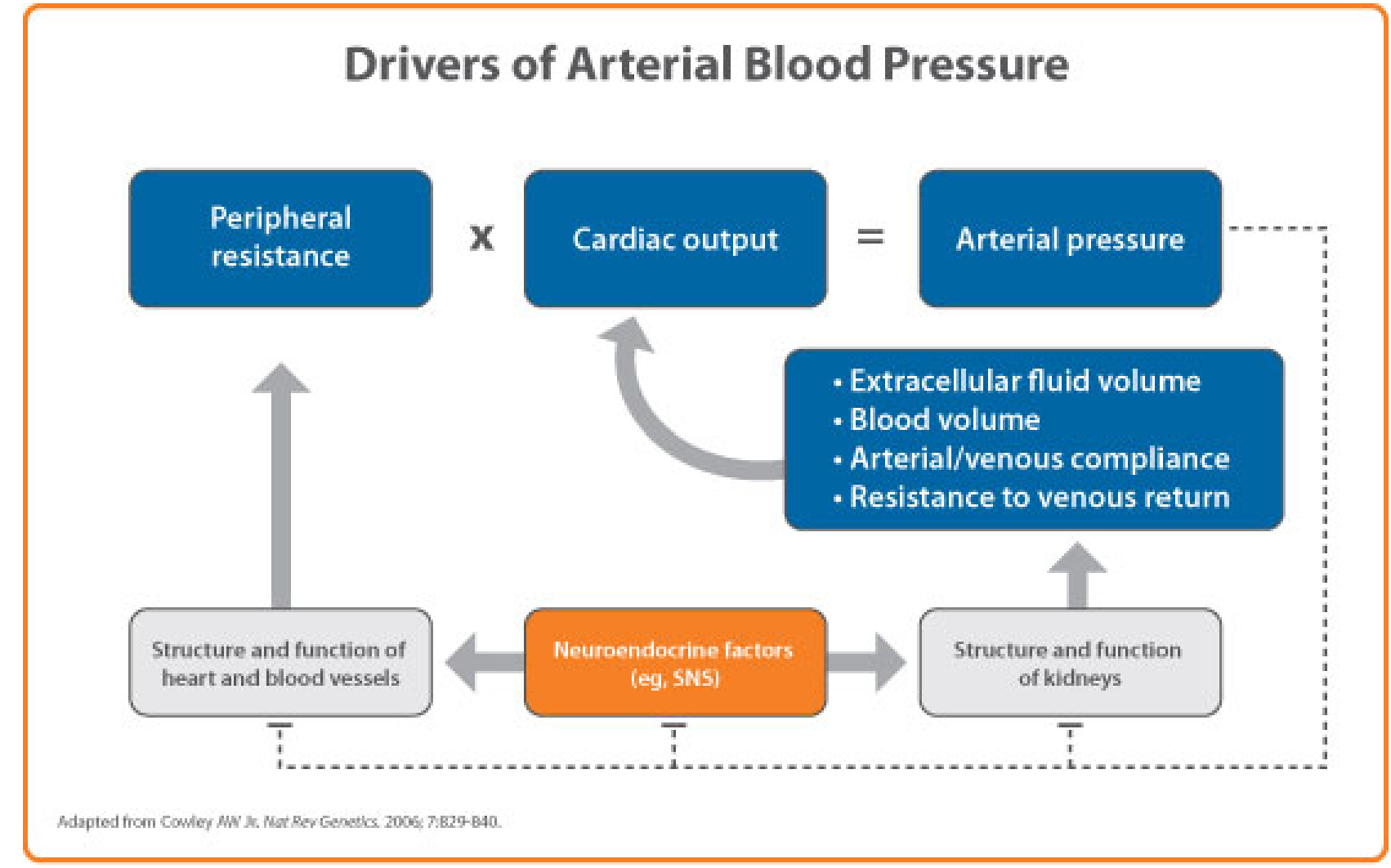

Figure 1. The relationship between mean arterial blood pressure (MAP), Cardiac output (CO) and total peripheral resistance (TPR)

\subsection{Criteria of Patients Selection}

All patients require infiltration LA injection for dental treatment (extraction) for maxillary teeth only have no history of compromised medical status, no recent use of antibiotics or analgesic drugs (in the last one week), nonpregnant or lactating females, non- smoker, non alcoholic, and agreed to participate in this study and signed the consent form.

\subsection{Study Design and Sample}

\subsubsection{Patient Grouping}

Patients included in this study were examined in two main situations: Pre- anesthetic situation included patients who require a dental treatment (tooth extraction ) and were seated on a dental chair for dental and clinical examination but before taking $\mathbf{L A}$ injection. This group consisted of a total number of (26) patients (8 M, $18 \mathrm{~F}$ ) with mean of age (20-40) years. Post- anesthetic situation: included patients who were taking LA Injection for dental treatments (tooth extraction). This group consisted of total number of (26) patients ( $8 \mathrm{M}, 18 \mathrm{~F}$ ) with mean age of (20-40) year.

\subsection{Drug Used}

\subsubsection{Local Anesthesia}

Local anesthetic cartridges $1.8 \mathrm{ml}$ (lidocaine -hamein $2 \%$ with 1:80,000 adrenalin) $\mathrm{MOH} /$ IRAQ were used to produce anesthesia by infiltration to maxillary teeth. [8] The anesthesia was administrated by the researcher only for those patients.
Materials and equipments used for collection, separation and storage of saliva

\begin{tabular}{|c|c|}
\hline Equipment & supplier \\
\hline Salivette & Sarstedt(Germany) \\
\hline Centrifuge 80 -1 TAPLETOP low speed & GSJ, China \\
\hline Micro pipette $10-100 \mathrm{ml}$ and tips & Jianan, China \\
\hline Eppendroff tube $1.5 \mathrm{ml}$ & China \\
\hline Rank for Eppendroff tube & China \\
\hline Refrigerator & Biofamily, Korea \\
\hline Freezer & Universal, Korea \\
\hline Cool box & China \\
\hline
\end{tabular}

\subsection{Salivary Sample Collection and Storage}

Unstimulated saliva was collected from patients before and after administration of local anesthesia. Patients were asked to rinse the mouth with $10 \mathrm{ml}$ of tap water to remove food debris then a simple cotton dental roll of specialized devices (Salivette) is sucked or chewed in patients mouth (cheek, floor of mouth, and over the tongue) for a 1 minute allowing the saliva to be absorbed and collected in the swab in an easy and hygienic fashion. All samples were collected between 9-11 am and should be clear of blood contamination. [9] Salivette then centrifuged at $3000 \mathrm{rpm}$ for $10 \mathrm{~min}$ and a clear fluid at the bottom of tube was placed in sterile eppendroff tube and stored at deep freeze _ $20 \dot{\mathrm{C}}$ until the time of analysis [10].

\subsection{Measuring Blood Pressure and Heart Rate}

The blood pressure and heart rate of patients were measured pre- and post administration of local anesthesia 
using an automatic blood pressure recorder (Rossmax, China). Blood pressure and heart rate recording were repeated immediately to obtain the mean reading [11].

\subsection{Human Chromogranin A Measurement}

Human chromogranin A was measured by ELISA kits (My BioSource, USA). The test is based on the quantitative sandwich enzyme immune- assay technique.

\subsection{Data Analysis}

The data obtained from this study was subjected to the statistical analysis including descriptive and analytic methods. For descriptive way the mean of variance was used, while one way analysis of variance and Duncan's Test for (intra and inter group comparison) matching with $5 \%$ level of significance. Paired t-test was used to compare between the study groups, while spearman test was used to estimate the possible correlation between the studying parameters.

\section{Results}

Table 3. Mean values of chromogranin A $(\mathrm{ng} / \mathrm{ml})$ pre and post administration of local anesthesia

\begin{tabular}{|c|c|c|c|c|c|c|c|}
\hline Parameters & $\begin{array}{c}\text { Pre-local anesthesia Mean } \\
\pm \text { SD }\end{array}$ & $\begin{array}{l}\text { Post-local anesthesia } \\
\text { Mean + SD }\end{array}$ & $\begin{array}{l}\text { Difference Mean } \pm \\
\text { SD }\end{array}$ & $\begin{array}{l}\text { 95\% CI of } \\
\text { difference }\end{array}$ & $\begin{array}{c}\text { T- } \\
\text { value }\end{array}$ & DF & P-value \\
\hline $\begin{array}{c}\text { ChromograninA } \\
\text { nglml }\end{array}$ & $580.33 \pm 130.42$ & $674.51+130.93$ & $-96.17 \pm 63.99$ & $-81.02-29.32$ & -4.396 & 25 & $<0.0001$ \\
\hline
\end{tabular}

Analysis was performed using paired $-t$ test

The hemodynamic effects of local anesthesia are shown in Table 4. The mean values of systolic and diastolic blood pressure were reduced after administration of local
A total of 26 patients participated in this study. Most of them were females (69.2\% females, $30.8 \%$ males). The mean age of samples was $22.5 \pm 6.68$ years for female and $30.5 \pm 3.5$ years for male with age range 20-40 years (Table 1 and Table 2).

Table 1. Distribution of the study samples

\begin{tabular}{|c|c|c|c|c|c|}
\hline Sex & Number & Percentage & P-value & DF & $\chi^{2}$ \\
\hline Male & 8 & (30.8) & & & \\
\hline Female & 18 & $(69.2)$ & & & \\
\hline Total & 26 & 100 & 0.7 & 2 & 0.556 \\
\hline
\end{tabular}

Analysis was performed by ANOVA test

Table 2. Descriptive statistic of study samples for both sexes

\begin{tabular}{|c|c|c|c|}
\hline Sex & Mean of age \pm SD & Minim & Maximum \\
\hline Females & $22.5 \pm 6.68$ & 20 & 40 \\
\hline males & $30.5 \pm 3.5$ & 20 & 40 \\
\hline
\end{tabular}

SD: standard deviation

The results of this study showed that the mean amount of chromogranin A in patients before taking local anesthesia was $(580.33 \pm 130.42) \mathrm{ng} / \mathrm{ml}$ while its amount was $(674.51+130.93) \mathrm{ng} / \mathrm{ml}$ in patients after taking local anesthesia (Table 3) with significant differences between them $(\mathrm{p}<0.05)$. anesthesia but with no significant differences ( $p>0.05$ ), while the pulse rate was increased after administration of local anesthesia with a significant differences ( $\mathrm{p}<0.05)$.

Table 4. The pre and post hemodynamic effects of local anesthesia

\begin{tabular}{|c|c|c|c|c|c|c|c|}
\hline Parameters & $\begin{array}{c}\text { Pre-local anesthesia } \\
\text { Mean } \pm \text { SD }\end{array}$ & $\begin{array}{c}\text { Post-local } \\
\text { anesthesia } \\
\text { Mean } \pm \text { SD } \\
\end{array}$ & $\begin{array}{l}\text { Difference Mean } \pm \\
\text { SD }\end{array}$ & $\begin{array}{l}\text { 95\% CI of } \\
\text { difference }\end{array}$ & T-value & DF & P-value \\
\hline $\begin{array}{l}\text { Systolic blood } \\
\text { pressure }\end{array}$ & $121.65 \pm 20.62$ & $120.96 \pm 22.19$ & $-1.69 \pm 17.96$ & $-4.56-9.94$ & 0.764 & 25 & 0.4 \\
\hline $\begin{array}{c}\text { Diastolic blood } \\
\text { pressure }\end{array}$ & $78.92 \pm 13.08$ & $77.76 \pm 16.75$ & $1.15 \pm 16.82$ & $-5.64-7.94$ & .350 & 25 & 0.7 \\
\hline $\begin{array}{c}\text { Pulse rate } \\
\text { Pulse/minute }\end{array}$ & $87.88 \pm 13.25$ & $89.65 \pm 13.19$ & $-2.76 \pm 8.09$ & $-8.03-1.50$ & -3.006 & 25 & 0.006 \\
\hline
\end{tabular}

Analysis was performed using paired t test.

The correlations between the studying parameters performed by pearson correlation and spearman test are shown in Table 5 which demonstrated inverse significant correlation between chromogranin A and systolic blood pressure with significant difference $\mathrm{p}<0.05$.

Table 5. The correlation between chromogranin A, systolic, diastolic blood pressure and pulse rate

\begin{tabular}{|c|c|c|c|}
\hline & Systolic & Diastolic & Pulse rate \\
\hline \multirow{2}{*}{ Chromogranin A } & $\mathbf{- 0 . 2 6}^{*}$ & $\mathbf{- 0 . 0 4}$ & $\mathbf{- 0 . 0 5}$ \\
\cline { 2 - 4 } & 0.02 & 0.7 & 0.6 \\
\hline
\end{tabular}

Note: upper bold fig (correlation coefficient r), lower figure (p-value), analysis were performed by pearson correlation test and spearman test

\section{Discussion}

The lignocaine-adrenaline combination is the most widely used local anaesthetic solution in dental practice. This combination is essential as adrenaline counteracts the known localized vasodilator effects of lignocaine in subcutaneous and submucosal vessels by causing vasoconstriction, thereby acting as a "chemical tourniquet" and thus decreasing the rate of systemic absorption from the site of injection and reducing the risk of anesthetic toxicity. Adrenaline increases the depth and duration of action. In addition, it provides haemostasis for surgical procedures. [12] Our study showed that a low dosage of adrenaline may produce significant hemodynamic effects particularly obvious hypotension combined with slight increase in pulse rate. Many hemodynamic studies were carried out on patients subjected to local anesthesia with vasoconstrictor. $[13,14,15,16]$ No significant changes were recorded in either blood pressure (systolic and diastolic) or heart rate. However, some authors suggested that such changes were dependent upon the injected vasoconstrictor dose. The hypotensive effect of epinephrine at subtherapeutic concentrations was evaluated in a series of reports by Yang et al., (2005). [17] Although both $\alpha$ - and $\beta$ adrenocepters are stimulated, $\beta 2$ vasodilator effect is most sensitive. Thus, in large doses, direct stimulatory effects on cardiac output plus potent vasoconstriction (particularly in precapillary resistance vessels of skin, 
mucosa, and kidney) produce a rapid increase in systolic arterial pressure. Diastolic pressure is affected less because of $\beta 2$ - receptor induced vasodilation in muscle beds (the characteristics physiologic distribution of the circulation associated with adrenaline), therefore the pulse pressure widens.

This results were also in agreement with many other studies like Bortoluzzi et al., (2010) [18] who explained results with regard to adrenaline and adrenergic system stimulation. It might be that adrenaline has both $\beta 1$ and $\beta 2$ activity. $\beta 1$ stimulation tends to cause an increase in blood pressure, whereas $\beta 2$ stimulation tends to decrease blood pressure, therefore it often does not dynamically increase blood pressure due in part to $\beta 2$ activity. A second possible explanation is that the hemodynamic alterations are usually short in plasma due to short adrenaline halflife which is approximately less than three minutes. In addition, when stimulated, the sympathetic nervous system primarily releases norepinephrine and secondarily releases epinephrine. The effects of both substances on blood pressure are limited [19,20].

The study of Haghighat et al., ( 2006) [21] showed a similar results and explained the increase of systolic blood pressure was probably due to stress (pre- administration of local anesthesia) while the lowering of systolic blood pressure after administration of local anesthesia(postadministration of local anesthesia) was probably due to disappearance of patients fear and stress after injection. Most of patients were more worried about injection than the treatment plan. During the injection process, the sight of dental syringe and apprehension of needle stick pain is considered a stressful condition to which in turn stimulates both $\alpha 1, \beta 2$ receptors in blood vessels at the site of injection. They initially cause vasoconstriction as $\alpha 1$ stimulation is predominant. However, subsequent biotransformation, reuptake and redistribution resulting low tissue level making $\beta 2$ action predominant with resulting vasodilation [22].

Another proposed mechanism for the occurrence is based on activation of the Bezold-Jarisch reflex (BJR). This reaction may be related to venous pooling caused by the sitting position and epinephrine-induced $\beta 2$-adrenergic effect and increased inotropy (adrenergic effect of epinephrine). Increased epinephrine levels may occur endogenously from decreased venous return and carotid baroreceptor stimulation as well as exogenously from epinephrine administered with the local anesthetic or in the irrigating solution. In this setting, a low-volume hypercontractile ventricle causes stimulation of intramyocardial mechanoreceptors ( $\mathrm{C}$ fibers) followed by an abrupt withdrawal of sympathetic outflow and an increase in vagal tone with resultant bradycardia and hypotension. [23] Similar results were obtained by Shaban et al, (2013) [24] and explained the increase in pulse rate after administration of local anesthesia due to the vasodilating effects of lidocaine .

The measurement of salivary chromogranin A (CgA) concentration has been developed as a method to evaluate psychological stress. The use of saliva rather than blood has obvious advantages including the ease of collection and noninvasiveness (painless) which is particularly important for pain and stress assessment. [25] Mitsuhata et al., (2013) [26] hypothysized that pre-treatment CgA values indicated the stress associated with dental treatment and post-treatment CgA values indicated relief from stress after the treatment with no significant correlation between them.

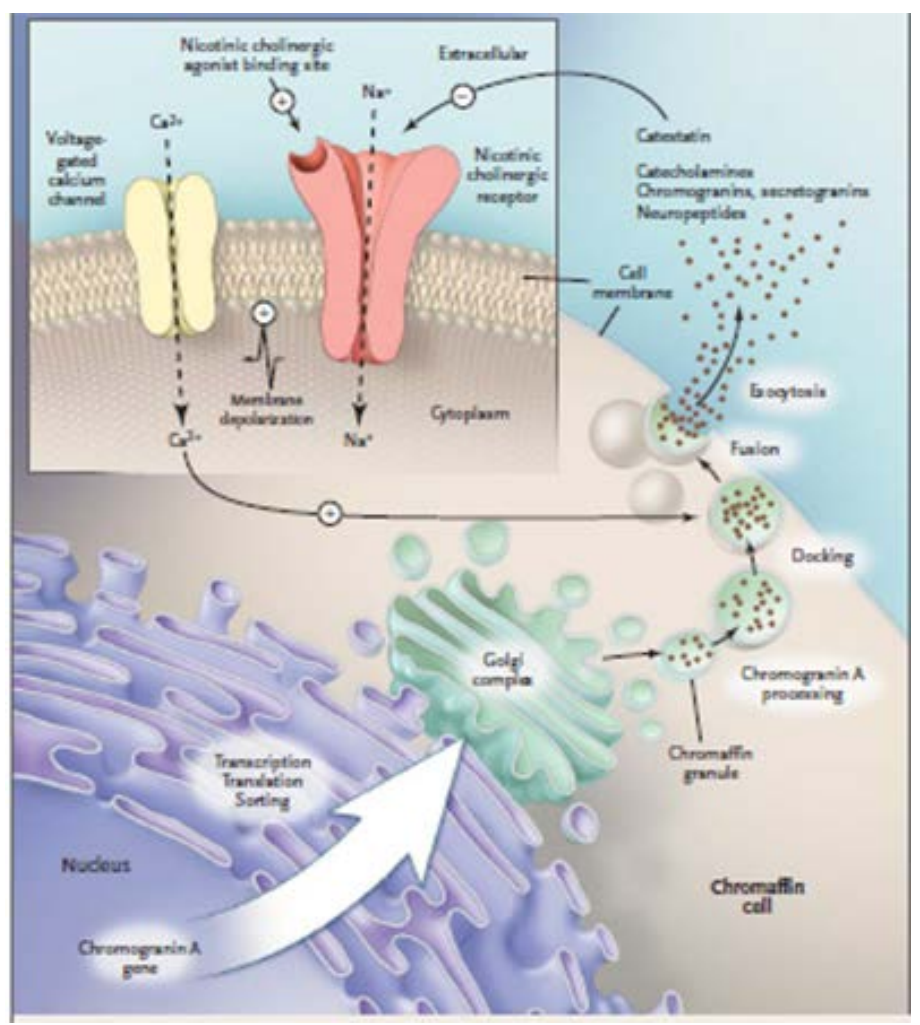

Figure 2. Catestatin role in regulation of catecholamine secretion

There was no previous study on the effects of local anesthetic in dentistry on salivary CgA. According to the results of this study higher amount of CgA after administration of local anesthesia was observed with a significant difference from patients before administration of local anesthesia. The possible explanation of this higher 
amount is that the injection pressure of local anesthesia causes pain due to several factors influence pressure including volume injected into tissue per unit time, permeability of the injected solution into bone, soft tissue, blood vessels, and effects of tissue pressure and stretching of the movable mucosa caused by volume of injected solution. [27] The pain and fear of pain are positively related to stress both during pain and in the anticipation of pain so that the fear of pain would be related to higher stress and pain intensity and to reduce placebo analgesia. [28] CgA is considered a biomarker of acute stress which causes activation of sympatho- adreno - medullary system and releases of CgA which has half life of 15-20 minutes. [29] The results of this study showed inverse correlation between CgA and systolic blood pressure and this could be explained by the fact that catestatin (a peptide fragment of the catecholamine secretory vesicle protein chromogranin A) is a potent inhibitor of exocytotic catecholamine secretion from PC12 and chromaffin cells. This peptide acts as a noncompetitive nicotinic cholinergic antagonist with characteristic inhibitory effects on nicotinic cationic (Na1, Ca21) signal transduction. Indeed, catestatin is more potent than substance $\mathrm{P}$ in inhibiting nicotine-induced secretion of catecholamines. [30] Catestatin is the first known endogenous compound able to inhibit in vitro catecholamine release from both chromaffin cells and noradrenergic neurons by acting as a non-competitive nicotinic cholinergic antagonist. [31] Angelone et al., (2008) [32] hypothesized that circulating levels of catestatin decreased in the plasma of patients with essential hypertension. Genetic ablation of the chromogranin A (Chga) gene in mice increases blood pressure and pretreatment of Chga-null mice with Cts prevents blood pressure elevation indicating a direct role of Cts in preventing hypertension. This is in agreement with the study of Tsigelny etal (2013) [33]. Catestatin inhibits the release of catecholamines from sympathoadrenal chromaffin cells by blocking the neuronal nicotinic cholinergic receptor which is the physiologic trigger for secretion [34] (Figure 2).

Catestatin also prevents the desensitization of catecholamine release from chromaffin cells that is induced by repeated nicotinic-agonist stimulation. Thus, catestatin may contribute to an autocrine negativefeedback mechanism that modulates catecholamine release within the sympathoadrenal system. Since excess sympathetic activity was implicated in the development of hypertension, a disturbance of the catestatin mechanism may be a contributing factor. O’Connor et al., (2002) [35] showed that the plasma catestatin level is diminished in patients with hypertension and even in normotensive persons at genetic risk for hypertension. The observation that a low catestatin level is correlated with increased adrenal epinephrine secretion and augmented pressor responses to sympathoadrenal stressors provides further evidence of a link between diminished catestatin and hypertension.

\section{References}

[1] American Academy of Pediatric Dentistry (2009). Guide line on use of local anesthesia for pediatric dental patient. Reference manual, 34 (6):183-90.
[2] Locker D, Liddell A, and Shopira D (1999). Diagnostic categories of dental anxiety: a population-based study. Behav Res Ther, 37:25-37.

[3] Helle KB (2004). The granin family of uniquely acidic proteins of the diffuse neuroendocrine system,comparative and functional aspect . Biol Rev (comb), 79:769-794.

[4] Vera J, Camoda M, and Machedo J (2011). Chromogranin A and $\mathrm{B}$ are key pprotiens in amine accumulation, but the catecholamine secretary pathway is conserved without them. FASEB, 18:Abstract

[5] Kanno T, Asada N, and Yanase H (1999). Salivary secretion of highly concentrated chromogranin A in response to noradrenalin and acetylcholine in isolated and per fused rat. submandibular glands. Exp Physiol, 84:1073-83.

[6] Obayashi K, Sato H, and Shimazzaki R (2008). Salivary chromogranin A: useful and quantitative biochemical marker of affective state in patients with any atrophic lateral sclerosis. Int Med, 47(21):1875-79.

[7] Saruta J, Tsukinoki K, and Sasaquri K (2005). Expression and localization of chromogranin A gene and protein in human submandibular gland. Cell Tissue Organs, 180(4).237-44.

[8] Malamed ST (2013). Technique of maxillary anesthesia In Malamed ST. Hand book of local anesthesia. $6^{\text {th }}$ edition, Mosby Elsevir Inc, New York, USA; p188-215.

[9] Kanegane K, Penha S, Borsatti M (2003). Dental anxiety in an emergency dental service. Rev Saude Publica, 37:786-792.

[10] Nater YM, Rohleder N, and Gaab J (2005). Human salivary alpha amylase reactivity in a psychosocial stress paradiagn. Int $\mathrm{J}$ Psychophysiol, 55(3):333-341.

[11] Meechan J, Cole B, and Welbury R (2001). The influence of two different dental local anesthetic solutions on the haemodynamic responses of children undergoing restorative dentistry: a randomized, single- blind, split- mouth study. $\mathrm{Br}$ Dent J, 190(9):502-504.

[12] Sotunmbi PT and Arotiba JT (2005). A manual of anaesthesia for dental and maxillofacial surgery. 1st ed., Ambod Publishers, Nigeria: 72-102.

[13] Meechan JG, Parry G, and Rattray DT (2002). Effects of dental local anaesthetics in cardiac transplant recipients. $\mathrm{Br}$ Dent $\mathrm{J}$, 192:161-3.

[14] Viana AM, Campos AC, and Morlin MT (2005). Plasma catecho lamine concentrations and hemodynamic responses to vasoconstrictor during conventional or Gow-Gates mandibular anesthesia. Oral Surg Oral Med Oral Pathol Oral Radiol Endod, 100:415-9.

[15] Cáceres MT, Ludovice AC, and Brito FS (2008). Effect of local anesthetics with and without vasoconstrictor agent in patients with ventricular arrhythmias. Arq Bras Cardiol, 91:128-33, $142-7$.

[16] Kameyama K, Watanabe S, and Kano T (2008). Effects of nasal application of an epinephrine and lidocaine mixture on the hemodynamics and nasal mucosa in oral and maxillofacial surgery. J Oral Maxillofac Surg, 66:2226-32.

[17] Yang JJ, Wang TY, and Wang J (2005). Marked hypotension induced by adrenaline contained in local anaesthetic. Laryngoscope 115(2):348-352.

[18] Bortoluzzi MC, Manfro R, and Nardi A (2010). Glucose levels and hemodynamic changes in patients submitted to routine dental treatment with and without local anesthesia. Clinics, 65(10):975978.

[19] Neal JM. (2003). Effects of epinephrine in local anesthetics on the central and peripheral nervous systems: Neurotoxicity and neural blood flow. Reg Anesth Pain Med, 28:124-34.

[20] Brown RS, Rhodus NL. (2005). Epinephrine and local anesthesia revisited. Oral Surg Oral Med Oral Pathol Oral Radiol Endod, 100:401-8.

[21] Haghighat A (2006). Hemodynamic effects of 2\% lidocaine with 1:80000 epinephrine in inferior alveolar block. Dent Res J, 3(1):47 .

[22] Chitre AP. (2010). Manual of local anesthesia in Dentistry. $2^{\text {nd }}$ ed. Jaypee Brothers Medical Publishers. pp. 3-90.

[23] Sia S, Sarro F, and Lepri A (2003). The effect of exogenous epinephrine on the incidence of hypotensive/ bradycardic events during shoulder surgery in the sitting position during interscalene block. Anesth Analog, 97: 583-588.

[24] Shaban B, Moradi M, and Nejat AH (2012). Hemodynamic Effect of $2 \%$ Lidocaine with 1:80,000 Epinephrine Infiltration in Maxillofacial Surgeries under General Anesthesia. JDM, 2(1):1625. 
[25] Shibata M, Kawai M, and Matsukura T (2013). Salivary biomarkers are not suitable for pain assessment in newborns. Early human development, 89:503-506.

[26] Mitsuhat C, Ohara Y, and Tachikake M (2012). Effectiveness of salivary chromogranin A as a stress index in young children during dental treatment. Pediatric Dental Journal, 22(2):163-169.

[27] Kudo M (2005). Initial injection pressure for dental local anesthesia: effects on pain and anxiety. Anesth Prog, 52(3):95-101.

[28] Lyby PS, Aslaksen PM, and Flaten MA (2010). Is fear of pain related to placebo analgesia? Journal of Psychosomatic Research, 68(4): 369-377.

[29] Koh DQ, and Koh GH (2007). The use of salivary biomarkers in occupational and enviremental medicine. Occup Environ Med, 64:202-210.

[30] Muhata SK, Mahata M, and Parmer RJ (1999). Desensitization of catecholamine release. The Journal of Biological Chemistry, 274 (5) :2920-2928.

[31] Imbrogno S, Garofalo F, Cerra MC (2010). The catecholamine release-inhibitory peptide catestatin (chromogranin A344-364) modulates myocardial function in fish. The Journal of Experimental Biology, 213: 3636-3643.

[32] Angelone T, Quintieri AM, Bhawanjit K (2008). The Antihypertensive Chromogranin A Peptide Catestatin Acts as a Novel Endocrine/Paracrine Modulator of Cardiac Inotropism and Lusitropism. Endocrinology, 149(10):4780-4793.

[33] Tsigelny IF, Kouznetsova VL, Biswas N.MahataSK, and OConnor DT (2013). Development of a pharmacophore model for the chatecholamine release- inhibitory peptide catestatin: Virtiual screening and functional testing identify novel small molecule therapeutics of hypertension. Bioorganic and Medicinal Chemistry, 21(18):5855-5869.

[34] Taupenot L, Harper KL, and Oconnor DT (2003). The chromogranin- secretogranin family. N Engl J Med, 348(12):113449.

[35] O’Connor DT, Kailasam MT, and Kennedy BP (2002). Early decline in the catecholamine release-inhibitory peptide catestatin in humans at genetic risk of hypertension. J Hypertens, 20:133545. 